\title{
Behandlungsfehler in der Psychotherapie: ein empirischer Beitrag zum Fehlerbegriff und seinen ethischen Aspekten
}

\author{
Irina Medau $\cdot$ Ralf J. Jox $\cdot$ Stella Reiter-Theil
}

Eingegangen: 7. Mai 2012 / Angenommen: 18. September 2012 / Online publiziert: 23. November 2012

(C) Springer-Verlag Berlin Heidelberg 2012

Zusammenfassung Behandlungsfehler in der Psychotherapie sind bisher kaum erforscht. Eine empirisch gestützte Kategorisierung von Behandlungsfehlern stellt einen ersten Schritt dar, sich evidenzbasierten ethischen Empfehlungen zum Umgang mit solchen Fehlern zu nähern. Zielsetzung dieser Arbeit ist es, dafür erste Grundlagen zu erarbeiten, die auf Erfahrungen von Praktikern Bezug nehmen. Nach einer systematischen Literaturrecherche wurden 30 semistrukturierte Interviews mit approbierten Psychotherapeuten unterschiedlicher Ausrichtungen (Schulen) geführt und anhand der qualitativen Inhaltsanalyse nach Mayring ausgewertet. Die beschriebenen, alltäglich auftretenden Behandlungsfehler konnten in technische, normative, Einschätzungs- und Systemfehler klassifiziert werden. Viele der technischen und Einschätzungsfehler wurden als reversibel angesehen; sie könnten sogar konstruktiv für die Behandlung nutzbar gemacht werden. Das Versäumnis, einen Fehler zu korrigieren, wurde als Hauptfehler betrachtet. Bei normativen Fehlern sei mit rechtlichen oder berufspolitischen Konsequenzen, aber auch mit Vertrauensverlust und Therapieabbruch zu rechnen. Für Systemfehler fühlten sich die befragten Therapeuten nicht verantwortlich; hier seien berufspolitische Änderungen nötig. Die Ergebnisse zeigen, dass die Befragten zu der Empfehlung tendieren, Psychotherapiepatienten in passender Form über Behandlungsfehler aufzuklären und in die entstehenden Konsequenzen einzubeziehen. Fazit: Psychotherapeuten äußern sich aufgeschlossen gegenüber einer transpa-

I. Medau, MSc $(\bowtie)$

Klinische Ethik Support \& Begleitforschung, Universitäre Psychiatrische Kliniken, Universitätsspital Basel, IBMB, Universität Basel

Schanzenstr. 13, 4056 Basel, Schweiz

E-Mail: Irina@ploytec.com

PD Dr. med. Dr. phil. R. J. Jox

Institut für Ethik, Geschichte und Theorie der Medizin, Ludwig-Maximilians-Universität

München, München, Deutschland

Prof. Dr. S. Reiter-Theil

Klinische Ethik Support \& Begleitforschung, Universitäre Psychiatrische Kliniken, Universitätsspital Basel, IBMB, Universität Basel, Basel, Schweiz 
renten, konstruktiven Fehlerkultur - eine wesentliche Voraussetzung für Fehlerprävention. Häufig resultiert erst durch die fehlende Korrektur eines (alltäglichen) Fehlers ein Behandlungsfehler, der Konsequenzen hat (z. B. Scheitern der Therapie). Um diesem entgegenzuwirken, zeichnet sich eine Befürwortung für eine passende Form der Patientenaufklärung über Fehler ab.

Schlüsselwörter Ethik in der Psychotherapie · Patientensicherheit · Empirische Fundierung des Fehlerbegriffs · Psychotherapeuten-Interviews · Qualitative Inhaltsanalyse

\title{
Treatment error in psychotherapy: an empirical contribution to the notion of error and its ethical aspects
}

\begin{abstract}
Aim Treatment errors in psychotherapy have rarely been a research topic until today. An empirically grounded categorisation of therapy error is a first step towards evidence-based ethical recommendations for the management of treatment errors. The following study aimed to provide foundations for the definition of treatment error in psychotherapy, which are based on the experiences of practitioners. Materials and methods After a systematic literature search, 30 semi-structured interviews with psychotherapists of different therapy directions (schools) were analysed using qualitative content analysis according to Mayring. Results The "normal" treatment errors described by the psychotherapists could be classified into technical, judgemental, normative and system errors. Many of the technical or judgemental errors were considered to be reversible; they could even be useful if used constructively in the treatment process. A missing revision of the error is considered the actual treatment error. Normative errors will most likely result in legal and professional consequences, loss of trust and cessation of the therapeutic relationship. System errors are met with the feeling of not being responsible; professional policies need to be adjusted. The results show that the interviewees tend to recommend disclosing errors in an appropriate way as well as including patients in the resulting consequences. Conclusion Psychotherapists appear open for a transparent, constructive error culturean essential condition for error prevention. Negative consequences (e.g., therapy failure) often only result by failing to revise the (everyday/normal) treatment error. To prevent this, therapists advocate appropriate error disclosure.
\end{abstract}

Keywords Ethics in psychotherapy · Patient safety ·

Empirical foundation of the concept of errors - Interviews with psychotherapists · Qualitative content analysis

\section{Hintergrund und Zielsetzung}

Im Rahmen der Bemühungen um Qualitätsmanagement, Patientensicherheit und Ethik nimmt auch in der Psychotherapie das Interesse am Thema Fehler zu; Probleme mit Aufklärung und Informed Consent werden, v. a. in der Verhaltenstherapie, häufig diskutiert [30].

Der Begriff „,medizinischer Fehler“ kam zwischen 1890 und 1935 auf, als eine medizinische Standardpraxis entwickelt wurde [7]. Ende des 20. Jahrhunderts wandte sich die Feh- 
lerdiskussion der Prävention zu. ${ }^{1}$ Ein Meilenstein war der Bericht des Institute of Medicine „To err is human“" [16].

Im Gegensatz zur Medizin gibt es in der Psychotherapie deutlich weniger Reflexion und empirische Forschung zu diesem Thema [15].

Die fehlende Auseinandersetzung mit dem Thema mag mit der Haltung Freuds zusammenhängen, der Fehler primär auf die Dynamik der Patienten/-innen zurückführte [18]. Eine erste Ausnahme stellte Willhelm Reich dar, der 1949 schon psychotherapeutische Behandlungsfehler beschrieb, insbesondere die Unfähigkeit von Psychotherapeuten, mit negativer Übertragung umzugehen. Seit den 1960er Jahren wurde vermehrt das Thema Schaden durch Psychotherapie diskutiert $[8,36]$, ebenso sexueller Missbrauch und Machtmissbrauch durch Therapeuten [33] sowie negative Therapieeffekte [10]. Heute geht man davon aus, dass 4-5\% aller Psychotherapien zu Verschlechterungen führen und bis zu 30\% erfolglos bleiben [9]. Welcher Teil davon mit Behandlungsfehlern im Zusammenhang steht, ist unbekannt.

Eine Schwierigkeit liegt in der unklaren Konzeption des Fehlerbegriffs - eine allgemein anerkannte Fehlerdefinition steht aus [12]. In der Fachliteratur finden sich unterschiedliche Definitionen und Klassifizierungen von Behandlungsfehlern: Haftungsrechtlich sind sie definiert als vermeidbarer Verstoß gegen den Standard angemessener Sorgfalt [2]. Offensichtliche Sorgfaltspflichten betreffen z.B. den Bruch des Abstinenzgebotes oder das Versäumnis, einen suizidalen Patienten auf seine Suizidabsichten hin zu befragen [39]. Eine Schwierigkeit dabei ist, dass nur nachweisliches und für Schäden ursächliches Fehlverhalten rechtlich erfasst wird [39].

Im Gegensatz zu technischen Disziplinen (z. B. Luftfahrt) ist die Feststellung eines Fehlers in der Psychotherapie nicht immer eindeutig möglich, sondern muss sich auf Standards oder Regeln, die von der Profession festgelegt und überwacht werden, beziehen. So werden im Managementhandbuch für Psychotherapeuten Fehler zusammenfassend definiert als unangemessene Durchführung von Diagnostik oder Therapie oder eine falsche Indikationsstellung, wobei gegen anerkannte Richtlinien bzw. aktuelle Standards verstoßen wird oder Grundregeln des therapeutischen Verhaltens dem Patienten gegenüber missachtet werden [2]. Die Komplexität der Psychotherapie und der Pluralismus der Therapiekonzepte verlangt eine differenzierte und praxisnahe Definition und Operationalisierung, die aber erst noch entwickelt werden muss [28].

Ein erster empirischer Zugang zum Thema Behandlungsfehler wurde 1979 von Bosk ${ }^{2}$ in einer Feldstudie eröffnet [4]. Für die Psychotherapie wurden verschiedene Kategorien von Fehlern vorgeschlagen [17]. Die Priorität sollte auf alltäglich auftretende Fehler statt auf (seltenere) juristische Verfehlungen wie etwa sexuellen Missbrauch gelegt werden [3]. So arbeitet Emmelkamp mit den Begriffen Wahl der falschen Technik und Verfahrensfehler [8]; bei Sponsel findet man eine Einteilung nach Therapiephase (z. B. Diagnostik- oder Katamnesefehler) bzw. Ursachen [35]. Kottler und Blau beschreiben Anfängerfehler und technische Fehler [17]. Hutterer-Krisch beschreibt Fehler in der Handhabung von Gegenübertragung, durch mangelnde Kenntnis der Kultur eines Patienten, mangelnde Achtung vor der Patientenautonomie, unzureichende Einfühlung, überholte Theorien oder Überforderung durch das Störungsbild [13]. Insgesamt ist die Literatur arm an empirisch gestützten Beiträgen, die sich nicht nur auf den Erfahrungshintergrund einzelner Autoren beschränken [22]. Die vorliegende Studie soll hierzu einen Beitrag leisten.

\footnotetext{
${ }^{1} 1988$ prägte Charles Perrow den Ausdruck ,normaler Fehler“ [27].

${ }^{2}$ Bosk, der oft als der Fehlerforscher schlechthin bezeichnet wurde, erarbeitete Fehlerkategorien für die somatische Medizin.
} 
Die folgenden Forschungsfragen stehen im Zentrum unserer Untersuchung: Wie klassifizieren und definieren Psychotherapeuten (PT) selbst Behandlungsfehler (BF)? Welches sind die häufigsten Fehler? Können Therapeuten eigene bzw. aus der Supervision bekannte Fehler beschreiben? Wie gehen sie mit Fehlern von Kollegen um? Welche moralischen Einstellungen haben sie zu Fehlern?

Ziel dieser Arbeit ist es, praxisorientierte Einsichten zum Thema Behandlungsfehler einer ethischen Diskussion zuzuführen. Dafür sollte der Fehlerbegriff mit alltagsrelevantem Inhalt angereichert werden. Durch die genauere Beschreibung von alltäglichen Fehlern sollen Empfehlungen angeregt werden, die einen fehlerfreundlichen Umgang fördern, gleichzeitig Schaden für den Patienten verhindern helfen und ggf. in künftige Leitlinien einfließen können. Weiterführende Überlegungen zum Umgang mit Fehlern in der Psychotherapie finden sich bei Medau et al. [25]. Im Interesse der Patienten und im Sinne einer ethischen Verantwortung von Psychotherapie ist eine vertiefte Beschäftigung mit dem Thema unerlässlich [30].

\section{Studiendesign und Untersuchungsmethoden}

Da es sich um ein kaum erforschtes Gebiet handelt, wurde das explorativ-qualitative Design einer Interviewstudie gewählt [24]. Auf der Basis eigener psychotherapeutischer Berufserfahrung in der Autorengruppe und einer Literaturrecherche (Datenbanken PubMed, PsychInfo) wurde ein semistrukturierter Interviewleitfaden [24] entwickelt, der die Themen Fehlerklassifikation und -definition, Umgang mit Fehlern und moralische Einstellung beinhaltet. Der Leitfaden wurde von Experten für qualitative Sozialforschung, Psychotherapie und Rechtsmedizin überprüft, in einer Vorstudie mit Hilfe von fünf Psychotherapeuten (PT) in Ausbildung erprobt. Hierbei zeigte sich, dass eine allgemein gehaltene Fehlerdefinition ([2] s. oben) nötig war, um die PT auf das Thema Behandlungsfehler (BF) einzustimmen. ${ }^{3}$

Als Studienteilnehmer wurden praktizierende PT aus dem Großraum Berlin ausgewählt, welche die anerkannten, erstattungsfähigen Psychotherapiemethoden anwenden, stationär und ambulant arbeiten (Cluster-Sampling mit Stratifizierung). Angefragt wurden insbesondere Supervisoren; durch die Vermittlung weiterer Kontakte konnten zusätzliche Interviewpartner gefunden werden (Snowballing). Fünf PT lehnten aus Zeitgründen die Teilnahme ab. Die Anzahl der Studienteilnehmer wurde nach dem in der qualitativen Forschung etablierten Kriterium der inhaltlichen Sättigung bestimmt, d. h. die Rekrutierung wurde beendet, sobald zusätzliche Interviews keinen Informationsgewinn mehr lieferten.

Die Interviews fanden in den Arbeitsräumen der PT statt, wurden alle von der gleichen Interviewerin durchgeführt (approbierte PT mit Methodenschulung in qualitativer Sozialforschung) und dauerten etwa $1 \mathrm{~h}$. Sie wurden akustisch aufgezeichnet, wörtlich transkribiert und anonymisiert. Mit der Anonymität wurde auch Sanktionsfreiheit für die PT garantiert. Die Transkripte wurden mit Hilfe der Software MAXqda 2001 nach der Methode der qualitativen Inhaltsanalyse gemäß Mayring ausgewertet [24]. Dieses Verfahren ist zwischen einer klassifikatorischen und sinnrekonstruierenden Vorgehensweise angesiedelt, wobei theoriegeleitet gearbeitet wird und die Textinterpretation auf Basis eines Katego-

\footnotetext{
${ }^{3}$ Nach einer vorangehenden ethischen Beratung durch die Ostdeutsche Psychotherapeutenkammer (OPK) wurde die ethische Vertretbarkeit der Studie geprüft: Der Interviewleitfaden und die Studieninformation für die Teilnehmer erfüllten die ethischen Richtlinien. Laut Ethikvotum der OPK wurde die Studie daraufhin von einem ausführlichen Ethikantrag befreit.
} 
Tab. 1: Informationen zu Interviewpartnern $(n=30)$. (Adaptiert nach [25], S.327)

\begin{tabular}{lll}
\hline Eigenschaft & Ausprägungen & Daten \\
\hline Geschlecht (n) & Männlich & 9 \\
Alter in Jahren & Weiblich & 21 \\
& Spanne & $28-70$ \\
& Mittelwert & 45 \\
& Median & 46 \\
Praktische Erfahrung in Jahren & Standardabweichung & 11 \\
& Spanne & $3-40$ \\
& Mittelwert & 18 \\
& Median & 18 \\
& Standardabweichung & 12 \\
\hline
\end{tabular}

rienschemas erfolgt. Gütekriterien wie Intercoderreliabiltät, Verfahrensdokumentation und argumentative Interpretationsabsicherung zeichnen die qualitative Inhaltsanalyse aus und wurden auch in unserer Studie angewandt. Die Unterkategorien wurden in einem Verallgemeinerungsprozess abgeleitet; die Grundlage für dieses Vorgehen bildete ein am Material entwickeltes (induktives) Kategoriensystem.

Für die weitere Analyse wurden mithilfe theoretischer Erwägungen deduktiv Hauptkategorien gebildet. Dafür dienten u. a. die von Bosk beschriebenen Fehlerkategorien [4]; sie konnten für die Psychotherapie als theoretisch gewonnene Oberkategorien angewendet werden. Zur Weiterentwicklung und Überprüfung des Kategoriensystems - und somit zur Erhöhung der Reliabilität - erfolgten ausführliche Diskussionen in Forschungskolloquien zur Medizin- und Gesundheitsethik bzw. Psychotherapieforschung. Weiterhin wurde die Validität durch eine ausführliche Verfahrensdokumentation abgesichert.

Nach ausführlichem Training führte ein unabhängiger Codierer (Diplompsychologe) einen zweiten Codierdurchgang an $40 \%$ des Materials durch, wobei sich ein Gesamt-Interraterreliabilitätskoeffizient von 0,93 ergab. Teile der Originalzitate werden zur Überprüfbarkeit der Kategorien im Folgenden präsentiert. In der Ergebnisdiskussion findet zusätzlich eine argumentative Interpretationsabsicherung statt.

\section{Ergebnisse}

Insgesamt nahmen 30 PT an den Interviews teil; davon waren 21 Psychologen, sieben Psychiater und zwei Sozialpädagogen. Die Geschlechterverteilung (Frauen - Männer ca. 2:1) sowie die Verteilung ärztlicher gegenüber psychologischen PT (ca. 1:3) entspricht etwa der allgemeinen Verteilung von PT [5]. Achtzehn waren zusätzlich in der Supervision tätig. Alle Therapeuten waren approbiert (Verhaltenstherapie, Tiefenpsychologie/Psychoanalyse, Gesprächstherapie); mehrere hatten zusätzliche Qualifikationen (z. B. Hypnose, Körpertherapie, Musiktherapie) (Tab. 1).

Frage zur eigenen Definition des Behandlungsfehlers

Die Mehrzahl der Befragten konnte der eingangs vorgegebenen Definition des BF zustimmen, konstatierten allerdings, diese müsse weiter ausdifferenziert und operationalisiert werden. Ein zentrales Ergebnis zur Frage, ob der Begriff „Behandlungsfehler“ als solcher angemessen sei, war, dass die Befragten weniger gern diesen Begriff als vielmehr Syno- 
nyme oder detaillierter beschreibende Komposita mit diesem Wortstamm benutzten: $z$. $B$. Lapsus, Methoden-, Flüchtigkeits-, Beziehungs- oder Diagnostikfehler. Insgesamt äußerten sich die Therapeuten unzufrieden mit dem Fehlerbegriff; sie bevorzugten Euphemismen wie Suchbewegung, Umweg, Irrweg, falsche Richtung, schwierige Situation, Schwierigkeiten im Prozess, Störfall oder ungünstiges therapeutisches Verhalten. Dies begründeten sie damit, dass solche Begriffe in einem Gespräch mit dem Patienten über einen BF eine (nützliche) Strategie darstellten.

Die Therapeuten distanzierten sich klar von sexuellen und anderen Übergriffen; diese seien keine ,alltäglichen“ BF und sie ordneten sie korrekt als Straftat ein. ${ }^{4}$ Mehr als 50\% der Therapeuten sprachen spontan von einem „klassischen Fehler“, wenn PT Eigen- oder Fremdgefährdung des Patienten nicht erkennen. Dieser Fehler wurde als der bedrohlichste angesehen, da er zum Patientensuizid führen kann, und in Abgrenzung zu den unten aufgezählten Fehlern als weniger alltäglich empfunden.

Insgesamt ließen sich vier Oberkategorien von Fehlerarten aufstellen, die durch weitere Unterkategorien spezifiziert werden (Abb. 1):

1. Technische Fehler betreffen Therapietechniken und können an Richtlinien und Standards festgemacht werden.

\section{Beispiele (Zitate aus Interviews):}

Unterkategorie Diagnostik: Es wird zu früh lostherapiert, ohne eine genaue Diagnostik zu machen, vielleicht ist die Diagnostik auch unvollständig (...) Also ich erlebe zwei Sachen, zum einen so eine Art Gießkanne, ohne im Vorfeld aufgrund der Symptomatik differentialdiagnostische Überlegungen anzustellen und gezielt Diagnosen zu machen zum Ein- oder Ausschluss (...), oder ich mache manche Diagnostikinventare nicht, obwohl die vielleicht wichtig wären. (T16 $\left.{ }^{5}\right)$

Unterkategorie veraltete Technik: Fehler hmm - ich glaube, generell was am häufigsten passiert, dass man Techniken anwendet, die veraltet sind und von denen man weiß, oder von denen aktuell ausgegangen wird, dass sie nicht wirken und dass trotzdem daran festgehalten wird. (T18)

2. Eine zweite Kategorie bilden die Einschätzungsfehler. Dazu zählten neben Fehleinschätzungen bei Indikationsentscheidungen auch falsche Einschätzungen der therapeutischen Beziehung.

Beispiele:

Unterkategorie Beziehung: Wenn die Beziehung, also das Arbeitsbündnis, noch nicht steht, also das wird oft zu unsensibel wahrgenommen: Gibt es das Bündnis wirklich, stimmt die Chemie? (T3)

Unterkategorie Indikation: Therapiefehler - also im Alltag betrifft das mehr Einschätzungen, prognostische Einschätzungen. Also z. B. bei Fällen, wo ich damals viel Zeit investiert habe, weil ich vielleicht dachte, ja, hier kann man was erreichen, würde ich heute sagen, dort konnte man mit unserer therapeutischen Methode und Energie nichts erreichen. (T20)

\footnotetext{
${ }^{4}$ Das Thema sexueller Übergriffe wird hier aus Raumgründen nicht diskutiert.

5 Identifikationsnummer des Interviews.
} 


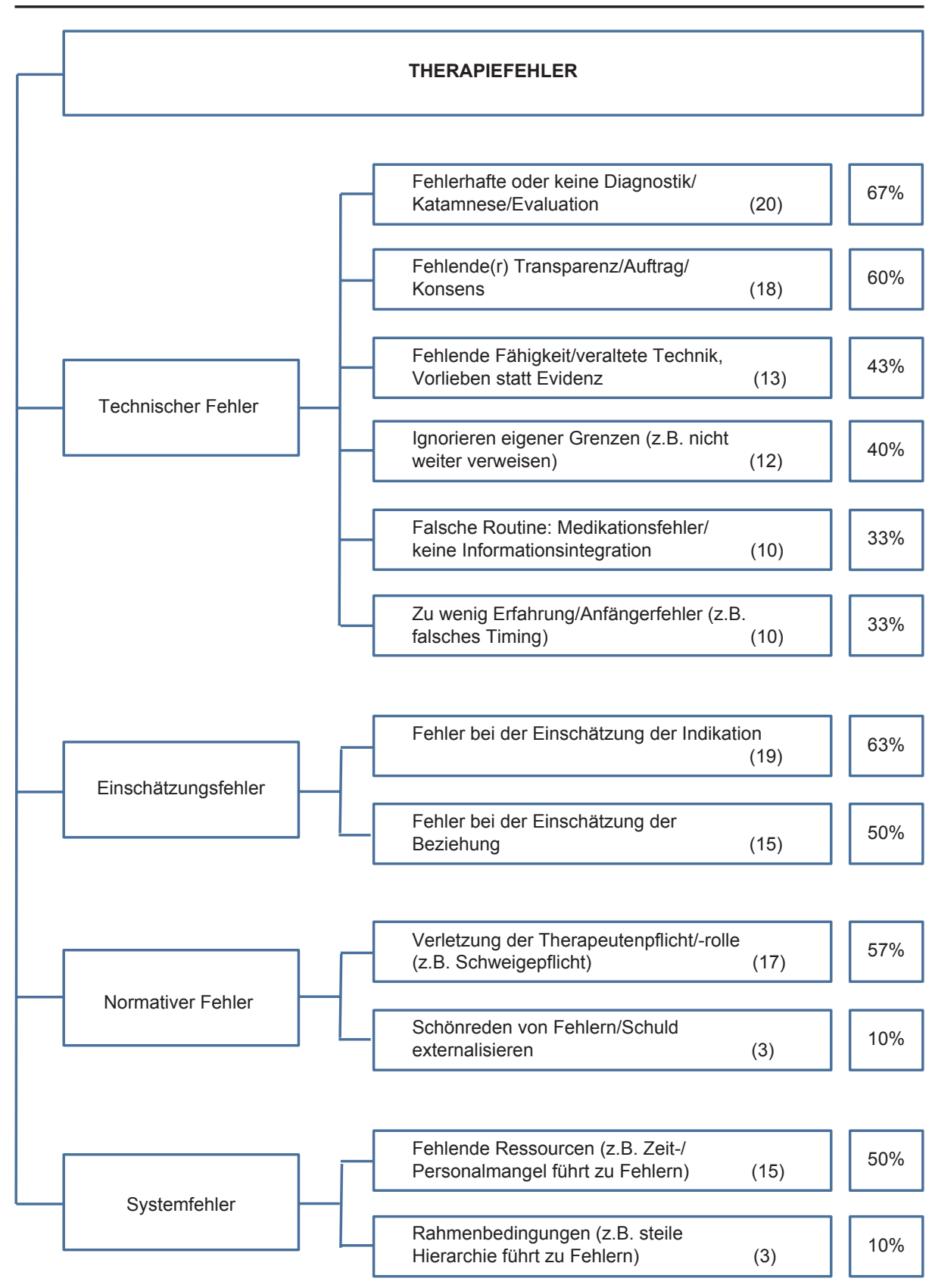

Abb. 1 Darstellung der vier Oberkategorien von Fehlern mit ihren Unterkategorien. Zahlen in Klammern geben die Zahl der Antworten an. Die Prozentzahlen geben den Anteil der Therapeuten an, die aus der Gesamtstichprobe $(100 \%)$ diese Fehler erwähnt haben. Mehrfachnennungen innerhalb einer Kategorie sind möglich 
3. Als normative Fehler im Sinne einer Abweichung von gesellschaftlich anerkannten moralischen, rechtlichen oder berufsrechtlichen Normen, wurden moralische Fehler wie Unaufrichtigkeit, die Abschiebung eigener Schuld auf andere und das Bagatellisieren bzw. Schön-Reden von Fehlern expliziert.

\section{Beispiele:}

Unterkategorie Schuld externalisieren: Einen klaren Misserfolg umdeuten, dem Patienten die Schuld geben (...), also manchmal hatte ich den Eindruck, wenn was schief gelaufen ist, dass dann die Eltern (bei Therapien von Kindern) dafür verantwortlich gemacht wurden, weil die ja nicht so gut kooperiert haben (...). (T12)

Unterkategorie Therapeutenpflicht: Was Therapiefehler betrifft, also eine Sache fällt mir noch ein, die zumindest in der Medizin noch immer dazu gezählt wird und meines Erachtens auch in unserem Arbeitsfeld wichtig ist - das ist unsere so genannte Aufklärungspflicht, der nicht immer in Gänze nachgekommen wird. In meinen Augen wäre das auch ein Fehler, wenn diesem so genannten und im Grundgesetz verbrieften informationellen Selbstbestimmungsrecht nicht nachgekommen wird (...). (T9)

Ich hab einen Fehler gemacht, einen Schweigepflichtfehler (...), das fand ich ganz fürchterlich. Da haben wir ganz viel in den Arztbrief geschrieben, der dann ans Jugendamt gegangen ist, und das Jugendamt hat natürlich den Eltern sofort Einsicht gewährt und da war die Jugendliche auch zu Recht ziemlich angefressen. (...) Da hatte ich auch nicht die Möglichkeit, damit souverän umzugehen, das anzusprechen, weil die Jugendliche schon entlassen war und nachstationär hat sie mich dann geächtet. (T12)

4. Diverse Systemfehler wurden beschrieben. Neben Fehlern, die aufgrund mangelnder Ressourcen (Zeitmangel, regionale Unterversorgung durch politische Deckelung von Praxiszulassungen) entstehen, wurden hier insbesondere organisatorische Rahmenbedingungen wie steile Hierarchien genannt. Diese Fehler werden im Rahmen des Systems in Kauf genommen; sie scheinen jedoch gleichzeitig von einer inneren Distanzierung und Verantwortungsdiffusion seitens der PT begleitet zu sein.

\section{Beispiele:}

Unterkategorie Rahmenbedingungen: Diese Crux der Befehlshierarchie, dass selbst wenn Therapeuten denken, das ist falsch, ich würde das anders machen, man den Willen des Oberarztes, wenn man nicht irgendwie subversiv veranlagt ist, durchführen muss. Das ist eine schwierige Sache, denn man fühlt sich ein bisschen so, wie wenn man wider besseres Wissen handelt und fühlt sich auch nicht so verantwortlich für das, was dann passiert. (T24)

Unterkategorie Ressourcen: Bezüglich Klinik wollte ich noch mal sagen - der Zeitdruck ist enorm, (...) ich finde das ist viel zu wenig vorgegeben, sich mal zu reflektieren und auszutauschen, ist immer alles so zwischen Tür und Angel und so hektisch, da passieren dann auch Fehler (...). (T15)

Insgesamt vertraten die Befragten, dass technische und Einschätzungsfehler häufig als reversibel angesehen würden, wirkliche BF entstünden demnach erst aufgrund von unterlassenen Korrekturen alltäglicher Fehler. 
Ich würde sagen, Behandlungsfehler entstehen wirklich erst daraus, wenn dann keine Rückkoppelung mit dem Patienten erfolgt (...), dann komm ich ja auf Holzwege und dann kommt's zu Behandlungsfehlern. (T20)

Der richtige Interaktionsfehler entsteht erst dadurch, dass man einen Fehltritt macht, und dann kommt nicht die Korrektur hinterher. (T23)

\section{Diskussion}

Für eine praxisrelevante Klassifizierung und Definition sind neben objektiven Daten auch subjektive Sichtweisen praktizierender Therapeuten wichtig. Die Interviewstudie zeigte teilweise überraschende Wahrnehmungen und Präferenzen der Befragten, die auch aus ethischer Sicht einer Diskussion wert sind.

\section{Begrifflichkeit und Kategorienbildung}

Insgesamt berichteten die Therapeuten freimütig von alltäglichen Fehlern. Der Begriff „Behandlungsfehler“ als solcher wurde zurückhaltend gehandhabt; die befragten PT machten Unterschiede bezüglich der Art von Fehlern und bevorzugten Euphemismen. Diese Sicht ist auch aus der Literatur ersichtlich [35] und könnte einerseits auf Banalisierung oder Tabuisierung des Themas hinweisen, welche die moralische Last des Begriffes abschwächt. Andererseits könnte dies auch auf eine konstruktive Fehlerkultur hindeuten. Eine weitere Erklärung könnte sein, dass der Begriff BF im deutschsprachigen Raum an juristische Prozesse denken lässt, zumal der verwandte Begriff Kunstfehler mit medialen Skandalberichten assoziiert wird. Allerdings hatte keiner der befragten PT selbst rechtliche Konsequenzen für einen Fehler erlebt.

Auch wenn, vielleicht mitbedingt durch eine fehlende Definition, noch keine genauen Zahlen von BF in der Psychotherapie bekannt sind, legen unsere Ergebnisse nahe, dass die beschriebenen ,alltäglichen Fehler“" als häufig wahrgenommen werden. Die in unserer Klassifikation gebildeten vier Oberkategorien dienen der Zusammenfassung der verschiedenen Unterkategorien; manche der Unterkategorien finden sich auch in anderen Fehlerklassifikationen $[8,13,17,35]$.

\section{Erste Oberkategorie: Technische Fehler}

Viele der beschriebenen alltäglichen Fehler konnten als technische Fehler subsumiert werden, diese Kategorie wird auch in der Literatur beschrieben [13, 35]. Die häufige Nennung von diagnostischen Fehlern ist wahrscheinlich darauf zurückzuführen, dass diese relativ leicht identifizierbar sind (z. B. falsches Rechenergebnis im IQ-Test). Ebenso sehen Therapeuten eine fehlende Therapieevaluation, inkl. Katamnese und Verlaufsevaluation, als augenfälligen Fehler an. Dies dürfte mit den in vielen Einrichtungen durchgeführten Qualitätssicherungsmaßnahmen zusammenhängen.

Obwohl technische Fehler noch am leichtesten wahrnehmbar erscheinen, spiegelt sich hier die Unsicherheit der Therapeuten wider, Fehler eindeutig als solche zu klassifizieren. Die Annahme, es müssten zur Bestimmung von Fehlern Leitlinien oder Standards zum Vergleich vorliegen, erschwert auch die Wahrnehmung von technischen Fehlern [4]. Leitlinien werden in der Psychotherapie kontrovers diskutiert [23]; verschiedene Psychotherapieschulen haben konkurrierende Standards. 
Zweite Oberkategorie: Einschätzungsfehler

Ein weiterer Fehlertyp wurde vom technischen Fehler unterschieden: der Einschätzungsfehler. Bosk [4] beschreibt medizinische Fehleinschätzungen bei der Auswahl therapeutischen Vorgehens. In der Psychotherapie sehen wir analog eine falsche Indikationsentscheidung. Eine weitere relevante Einschätzung ist die (Fehl-)Einschätzung der therapeutischen Beziehung. Im Unterschied zum technischen Fehler scheinen beim Einschätzungsfehler subjektive Komponenten eine größere Rolle zu spielen. Obwohl standardisierte Instrumente und Vorgehensweisen vorhanden sind, gilt z. B. die Indikationsentscheidung als ,nicht in idealer Weise beantwortbar“", da sie zusätzlich auf nicht überprüften Annahmen, individuellen praktischen Erfahrungen, Experten- und Kollegenmeinungen sowie Alltagswissen basiert [22]. Hier ist das „klinische Urteil“, aber auch die moralische Urteilskraft gefordert - komplexe Kompetenzen, die für die Psychotherapie noch zu analysieren sind.

Fehler bei der therapeutischen Beziehung werden bereits von anderen Autoren beschrieben $[3,13,35]$. Anhand der Antworten unserer Befragten zeigte sich, wie sehr PT für die hohe Relevanz der therapeutischen Beziehung sensibilisiert sind. Die Bedeutung der therapeutischen Beziehung für den Therapieerfolg wird durch neuere Psychotherapiestudien nur begrenzt gestützt [23].

Zunehmend operationalisierte Vorgehensweisen zur Erfassung von Therapiebeziehung und Indikation, die jedoch in der Praxis selten angewendet werden, erlauben es, hier auch von technischen Fehlern zu sprechen. Die Befragten unterschieden jedoch zwischen diesen zwei Fehlerarten; es ist fraglich, ob Beziehung und Indikation wirklich als reine Fragen der „Technik“ angesehen werden können. Beim Einschätzungsfehler spielen Empathie und persönliche Empfindungen eine Rolle; technische Fehler beziehen sich deutlicher auf professionelles Wissen. Daher scheint uns eine eigene Kategorie - Einschätzungsfehler - gerechtfertigt.

\section{Verkennen von Suizidalität}

Das fehlerhafte Verkennen von Suizidalität beim Patienten wurde nicht auf mangelnde Kompetenzen, sondern auf falsche Einschätzung zurückgeführt. Daher kann dieser ,klassische Fehler" den Einschätzungsfehlern (s. u.) zugeordnet werden. Die Einschätzung von Suizidalität bei psychisch kranken Menschen stellt eine der schwierigsten Aufgaben des Berufes dar, und ein Verkennen von Suizidalität ist der wohl am meisten gefürchtete Fehler. Man könnte vom therapeutischen ,Damoklesschwert“" sprechen; insbesondere das deutliche Bewusstsein über diesen potentiellen Fehler unterscheidet ihn von den anderen beschriebenen Fehlern. Hier sind neben Therapeutenvariablen Patienten- und Umweltvariablen besonders wichtig, was eine Einstufung als Einschätzungsfehler stützt.

In der Literatur wurden häufig Fehler im Umgang mit suizidgefährdeten Menschen beschrieben [13, 34], die aus heftigen Gegenübertragungen resultierten und zu falschen Reaktionen führten [13]; auch Übertragungsfehler können als Einschätzungsfehler klassifiziert werden. Bei diesem Fehler steht der Wert des menschlichen Lebens im Raum; die Konsequenz aus dem Fehler (Tod) ist hier irreversibel und der wohl größte Schaden, der einem Patienten widerfahren kann. Dieser Fehler wird moralisch nach seinen Folgen beurteilt. Die Relation zwischen negativen Folgen und Fehlern führt zur nächsten Unterscheidung. 
Fehler vs. Misserfolg

Hoffmann et al. [12] definieren Misserfolge als die unzureichende bzw. fehlende Erreichung angestrebter Ziele, und Therapieschäden als unerwartete, anhaltende und für den Patienten erheblich nachteilige Auswirkungen der Therapie. Im Unterschied dazu muss bei einem BF nicht immer ein Misserfolg oder Schaden vorliegen. Auch Bosk beschreibt, dass sowohl technische als auch Einschätzungsfehler häufig behoben werden können [4]. Gerade in einer Psychotherapie kann die Einübung fehlertoleranter Verhaltensweisen eine sinnvolle Strategie sein, um soziale Kompetenz und Stresstoleranz des Patienten zu fördern [17]. Ähnlich betonten unsere Befragten, dass technische und Einschätzungsfehler meist nicht endgültig seien; sie könnten durch richtige Handhabung korrigiert werden. Durch Bezeichnungen wie „Suchbewegung“, „Irrweg“ oder „Umweg“ könne man Patienten gut klar machen, dass in der Therapie manchmal Fehler passierten, die dann korrigiert werden müssten. Bei massiertem Auftreten oder mangelnder Bearbeitung der Fehler führten sie jedoch zu Verschlechterungen oder zum Scheitern. Ein Bewusstsein für dieses Problem könne die Fehlerprävention fördern. Schlussfolgernd stelle aber gerade die Unterlassung einer solchen Fehlerkorrektur den eigentlichen BF dar, der dann die ganze Therapie zunichte mache [36]. Wir sind hier mit einer deutlichen Tendenz konfrontiert, BF therapeutisch positiv nutzen zu wollen und womöglich als wenig gravierend für den Patienten zu bewerten.

Eine Schwierigkeit bei der Interpretation der vorliegenden Ergebnisse ist die in der Literatur mehrfach beschriebene Fehleinschätzung von Therapeuten bezüglich ihrer (schlechten) Therapieverläufe, bzw. ihre Tendenz, die eigene Arbeit positiver wahrzunehmen als sie ist [31]. Diese Voreinstellung könnte auch in Bezug auf eigene Fehler vorliegen und zu einer verzerrten Darstellung führen. Die eher positive Einstellung gegenüber Fehlern bzw. die Betonung der Reversibilität von Fehlern können damit teilweise erklärt werden. Einer solchen Voreinstellung könnte durch gezielte Sensibilisierung begegnet werden.

\section{Dritte Oberkategorie: Normative Fehler}

Normative Fehler umfassen ein Verhalten, das in Abgrenzung zu den o. g. Fehlern und ähnlich wie in der Medizin [4] häufig als moralisch verwerflich empfunden wird. Explizite Normen bezüglich der Psychotherapeutenrolle wurden für die Psychotherapie, z. B. im Rahmen von Berufsordnungen oder gesetzlichen Regelungen formuliert [13, 23, 32, 38]; moralische Normen sind hier jedoch oft implizit oder zumindest ungenügend expliziert [30] und ein Bedürfnis nach Orientierung ist ersichtlich [37]. Aufgrund des Pluralismus, der die Psychotherapie und ihre impliziten oder expliziten Wertvorstellungen prägt, sind normative Fehler oft nur schwer erkennbar [28], sofern sie nicht eindeutige Verfehlungen gegen etablierte Normen wie das Recht darstellen (z. B. Bruch der Schweigepflicht). Die Befragten nannten Fehler dieser Art daher häufig unter Vorbehalt und betonten die unterschiedlichen Haltungen verschiedener Schulen, die eine Orientierung erschweren. Ein interessantes Ergebnis ist, dass sich diese angenommenen Unterschiede nicht in den Ergebnissen widerspiegelten. Obwohl ein Pluralismus der Schulen herrscht (z. B. bzgl. Grad der Abstinenz), scheinen die Einstufungen als normative Fehler schulenübergreifend zu sein.

Wie aus anderen Studien bekannt [9, 19, 20, 29, 30], wurde mangelnde Aufklärung des Patienten auch in der aktuellen Stichprobe als einer der häufigsten Fehler eingeschätzt. Die Aufklärungspflicht umfasst die Indikation, Art der Behandlung, den Therapieplan, Behandlungsalternativen, Behandlungsrisiken und Rahmenbedingungen vor Beginn und während der Behandlung [23]. Bezüglich der konkreten Handhabung der Aufklärung wurden allerdings sehr unterschiedliche Haltungen und auch Unsicherheiten deutlich. 
Bei normativen Fehlern gilt, dass mit rechtlichen und berufsrechtlichen Konsequenzen, aber auch mit Vertrauensverlust und damit dem Therapieabbruch des Patienten zu rechnen ist. Um als Fehler zu gelten, müssen diese Verstöße in gewisser Weise „ungewollt“ sein. Absichtliche bzw. bewusst in Kauf genommene Verletzungen von Berufspflichten (z. B. sexueller Kontakt mit Patient) fallen nicht darunter - dies belegt die klare Abgrenzung zur Bezeichnung als Fehler durch die Befragten.

In diesem Zusammenhang ist es aufschlussreich, sich mit der Unsitte des Schönredens von Misserfolgen oder des Externalisierens von Schuld zu befassen; diese Praxis haben wir als normativen Fehler gekennzeichnet, weil gegen moralische Werte und Normen wie z. B. Ehrlichkeit verstoßen wird. Ebenso verstößt es gegen das ethische Prinzip der „Integrität", wenn für das Misslingen einer Therapie trotz eindeutiger BF ausschließlich oder vorwiegend mangelnde Motivation oder Persönlichkeitsmerkmale des Patienten als Erklärung herangezogen werden [21]. Ein unrealistisches Ideal der Fehlerfreiheit kann dazu beitragen, die Schuld bei Anderen zu suchen. Selbstschutzgründe können gegen eine Fehleranalyse sprechen und dazu führen, dass ausbleibende oder unzureichende Erfolge, mangelnde Motivation oder Persönlichkeitsmerkmale des Patienten zur Entschuldigung herangezogen werden $[6,17]$; dies geschieht nicht unbedingt bewusst.

\section{Psychotherapeutengesetz und Berufsordnung}

Wie können die vorliegenden Ergebnisse vor dem Hintergrund aktueller Regelwerke eingeordnet werden? Im deutschen Psychotherapeutengesetz ist geregelt, dass bei schwerwiegenden beruflichen Fehlern Disziplinarbestimmungen sowie berufsständische, gesetzliche oder verwaltungsrechtliche Regeln angewendet werden können. Fehler können insbesondere im Rahmen des Zivilrechts als Pflichtverletzungen geahndet werden [32].

Die American Psychological Association der USA hat bis heute die am besten ausgearbeiteten berufsethischen Prinzipien; ethische Verstöße (u. a. Fehler) werden hier geregelt $[1,13]$. Der Schweizer Psychotherapeutenverband empfiehlt PT das Eingestehen begangener Fehler und die Aufarbeitung mit dem Patienten. Beschwerden werden durch die Standeskommission geregelt, die ggf. eine Klage und Sanktionen veranlasst [38]. Der österreichische Berufskodex beschäftigt sich explizit mit Fehlern, der Ethikausschuss des Psychotherapiebeirates bietet hierfür einen Beschwerdeausschuss an ${ }^{6}$ [13].

Interessant ist insbesondere die Norm einer persönlichen und fachlichen Kompetenz sowie die Orientierung an fachlichen und wissenschaftlichen Standards. Folgt man dieser Norm, schafft dies eine Grundlage für das Erkennen technischer Fehler (Verstoß gegen Standards) und kann gleichzeitig als ethische Verpflichtung zur Vermeidung dieser Fehler betrachtet werden.

Vierte Oberkategorie: Systemische Fehler

Im Gegensatz zum individuell verursachten Fehler haben laut Fehlerforschung [26] die meisten Fehler eine systemische Komponente, z. B. unzureichende Arbeitsbedingungen und systembedingte Verhaltensmuster. Hierbei zählen auch eine geringe Psychotherapeutendichte, eine mangelnde Erreichbarkeit sowie eine Therapieübernahme durch nicht psychotherapeutisch qualifizierte Ärzte. Systemfehlern begegnen die befragten Therapeuten mit dem Gefühl

\footnotetext{
${ }^{6}$ Vergleichbar zu medizinischen Einrichtungen erscheint hier die Beschwerde- und Schlichtungsstellenkultur auch für die Psychotherapie gut etabliert.
} 
der Nicht-Verantwortlichkeit, Ohnmachtsgefühlen bzw. der Forderung nach Veränderungen auf höherer Ebene. Diese Thematik erfährt zunehmend Beachtung [3, 13, 30].

\section{Erheben und Erfassen von Fehlern - Fehlerprävention}

Bereits der Begriff „Fehlerkultur“ weist auf die Überwindung des Fehlereliminierenden Perfektionismus und der Null-Fehler-Toleranz hin [3]. In der Psychotherapie scheint ein eigenes Verständnis des BF erst in Ansätzen vorhanden zu sein. Auch ein kausal strukturierter technischer Rahmen, wie z.B. in der Luftfahrt, der eine eindeutige Fehlerklassifikation erlauben würde, fehlt. Fehleranalyse und ein Rückmeldesystem sind für einen konstruktiven und lernfördernden Umgang mit Fehlern wesentlich [3]. Hierfür kann die Technik als Ausgangspunkt der modernen Fehlerkultur genutzt werden. Die Erfassung von Beinahe-Fehlern oder Fehlern, die nicht zu einem Schaden geführt haben, hat in der somatischen Medizin (und zuvor in der Luftfahrt) bereits gute Ergebnisse gebracht [14, 16]. So könnten auch in der Psychotherapie anonyme Meldesysteme wie das Critical Incident Reporting System (CIRS) helfen. Erste Ansätze in diese Richtung gibt es bereits, z. B. das KÖDOPS, ${ }^{7}$ eine Software zur Falldokumentation und -sammlung aus der klinischen Praxis, die zur Planung und Qualitätssicherung von Psychotherapie (tiefenpsychologisch und analytisch) genutzt werden kann und u. a. Fehler dokumentiert.

Zur Prävention von Fehlern könnte weiterhin die Analyse (negativer) Verläufe in der Psychotherapie dienen; allerdings wären dazu entsprechende Leitlinien zur Definition der Abweichung notwendig. Ein Problem von Monitoringsystemen für Therapieverläufe ist die praktische Untauglichkeit der Verfahren. Auch für ein Monitoring durch äußere Stellen fehlen praktikable Konzepte.

Das Thema BF sollte zum festen Bestandteil der Psychotherapeuten-Ausbildung gemacht werden, ebenso wie die Auseinandersetzung mit ethischen Richtlinien der Berufsverbände. Zusätzlich könnten Maßnahmen wie eine persönliche Anerkennung (Unterschrift) ethischer Grundsätze bei Berufseintritt die existierenden ethischen Richtlinien stärker ins Bewusstsein rücken. Auch die Nutzung dieser Definition zur Erstellung von Leitlinien könnte einen ethisch vertretbaren und therapiefördernden Umgang mit BF ermöglichen. Ethische Empfehlungen wie die Offenlegung von Fehlern gegenüber den Patienten werden bereits in der Literatur diskutiert $[11,13]$. Weitere empirische Studien und Expertenmeinungen sind nötig, um valide Fehlerkategorien sowie eine belastbare Definition zu entwickeln. Insbesondere müsste aber bei der individuellen Sensibilisierung für das Thema und der Weiterbildung für ethische Aspekte in der Psychotherapie angesetzt werden.

\section{Einschränkungen}

In der regional begrenzten Stichprobe wurde die Befragung auf die Psychotherapeutensicht sowie auf Selbstauskünfte beschränkt. Neben sozial erwünschten Antworttendenzen könnte auch ein Teilnahme-Bias wirksam sein, falls dem Thema gegenüber eher aufgeschlossene Kollegen teilnahmen (fünf Absagen ggü. 30 Zusagen). Weiterhin einschränkend könnte für die Validität eine unzureichende Wahrnehmung von Fehlern und deren Auswirkungen sein. Eine breit angelegte Überprüfung der Kategorien im Rahmen einer repräsentativen Studie

\footnotetext{
${ }^{7}$ Kölner Dokumentationssystem für Psychotherapie und Traumabehandlung, beinhaltet u. a. auch ein Fehlermeldesystem, http://www.koedops.de/. Zugegriffen: 5. Mai 2012.
} 
über Fehlertypen und -häufigkeiten ist empfehlenswert. Zusätzlich sollte die Patientensicht erfragt werden. Abschließend kann festgehalten werden, dass die große Offenheit und das Interesse der Therapeuten gegenüber dem Thema in einem erstaunlichen Kontrast zur kaum erkennbaren Diskussion des Themas innerhalb der Psychotherapeutenzunft, z. B. in der Weiterbildung, stand. Hier besteht Nachholbedarf, dem die Institute im Zusammenhang mit einer kompetenten Berücksichtigung von ethischen Fragen in den Curricula entsprechen könnten.

\section{Fazit für die Praxis}

1. Die Therapeuten konnten sich mit der vorgegebenen Definition für BF (Behandlungsfehler) als unangemessene Durchführung von Diagnostik oder Therapie bzw. falsche Indikationsstellung identifizieren. Differenzierend nannten sie ungewollte Abweichungen von professionellen Standards sowie Fehleinschätzungen, die ohne Korrektur zum endgültigen Behandlungsfehler werden. Folgende alltägliche Fehler wurden unterschieden: technische Fehler, Einschätzungsfehler, normative Fehler und Systemfehler. Anstelle des technischen Fehlers wurden Alternativbegriffe (z. B. Suchbewegungen) bevorzugt. Charakteristisch für die Psychotherapie sind Einschätzungsfehler, deren Basis praktische Erfahrungen, Meinungen sowie Alltagswissen bilden. Beispielhaft für eine alltägliche normative Verfehlung war das Schönreden von Fehlern. Politisch interessant sind durch Einzelne nur geringfügig beeinflussbare Systemfehler, die Veränderungen auf höherer Ebene erfordern.

2. Nicht alle Fehler sind vermeidbar; entscheidend ist daher - auch für den Patienten der angemessene Umgang mit Fehlern. Fehler sollten gegenüber Patienten in passender Form angesprochen werden, gerade technische und Einschätzungsfehler gelten dann als häufig reversibel. Als eigentlicher Behandlungsfehler wird eine fehlende Korrektur des Fehlers angesehen.

3. Präventiv könnten bereits bekannte Fehler genutzt werden. Dazu benötigt werden (ähnlich wie in der somatischen Medizin): Frühwarnsysteme, Einrichten von Melderegistern, bessere Vermittlung einer professionellen Haltung und Selbstkontrolle. Reflexion und Umgang mit Behandlungsfehlern sollten im Zusammenhang mit ethischen Aspekten in der Aus-, Weiter- und Fortbildung zur Psychotherapie bei Ärzten und Psychologen vermittelt werden.

Danksagung Wir danken allen, die bei der Forschungsarbeit mitgewirkt haben; insbesondere den Psychotherapeuten, die für die Interviews zur Verfügung standen, dem Kolloquium zum Basler PhD-Programm Medizin- und Gesundheitsethik für kontinuierliche interdisziplinäre Diskussionen, dem Forschungskolloquium der Kinder- und Jugendpsychiatrischen Universitätsklinik Basel für konstruktive Rückmeldung, ebenso den externen Experten, insbesondere Prof. Silvia Schneider, Bochum, und Prof. Christina Schröder, Leipzig.

Interessenkonflikt Die Autoren geben an, dass keine Interessenkonflikte bestehen.

\section{Literatur}

1. American Pschological Association (1992) Ethical prinicples of psychologists and code of conduct. Am Psychol 47:1597-1611 
2. Behnsen E, Bell K, Best D, Gerlach H, Schirmer H-D, Schmid R (2000) Management-Handbuch für die psychotherapeutische Praxis. Decker, Heidelberg

3. Bienenstein S, Rother M (2009) Fehler in der Psychotherapie: Theorie, Beispiele und Lösungsansätze für die Praxis. Springer, Wien

4. Bosk CL (1981) Forgive and remember: managing medical failure. The University of Chicago Press, Chicago

5. Bundesärztekammer (KBV) (2003) Psychotherapeuten und Therapieverfahren und Geschlechterverteilung. Onlinedokument. Bundesärztekammer. http://www.psychogen.de/data/pool/2052813798.pdf. Zugegriffen: 5. Mai 2012

6. Dörner D (1989) Die Logik des Misslingens. Strategisches Denken in komplexen Situationen. Rowohlt, Berlin

7. Dupré K (1989) Ärztliche Behandlungsfehler. Geschädigte Patienten und ihre Rechtsansprüche. Jopp, Wiesbaden

8. Emmelkamp PMG (1988) Misserfolge in der Verhaltenstherapie. In: Kleiber D, Kuhr A (Hrsg) Handlungsfehler und Misserfolge in der Psychotherapie. Beiträge zur psychosozialen Praxis, Tübinger Reihe, Nr. 8. Deutsche Gesellschaft für Verhaltenstherapie, Tübingen, S 34-44

9. Fischer F (2007) Evidenzbasierte Psychotherapie? Wenn, dann schon richtig. Report Psychol 10:453-456

10. Grawe K, Donati R, Bernauer F (1994) Psychotherapie im Wandel: Von der Konfession zu Profession. Hogrefe, Göttingen

11. Greenson R (2000) Technik und Praxis der Psychoanalyse. Klett-Cotta, Stuttgart

12. Hoffmann SO, Rudolf G, Strauss B (2008) Unerwünschte und schädliche Wirkungen von Psychotherapie. Eine Übersicht mit dem Entwurf eines eigenen Modells. Psychotherapeut 53:4-16

13. Hutterer-Krisch R (2007) Grundriss der Psychotherapieethik. Praxisrelevanz, Behandlungsfehler und Wirksamkeit. Springer, Wien

14. Kaufmann M, Ständer S, von Below G, Brunner HH, Portenier L, Scheidegger D (2002) Computerbasiertes anonymes Critical Incident Reporting: ein Beitrag zur Patientensicherheit. Schweiz Ärztez $83: 2554-2558$

15. Kleiber D (1988) Handlungsfehler und Misserfolge in der psychosozialen Praxis: Probleme im Umgang mit komplexen Systemen. In: Kleiber D, Kuhr A (Hrsg) Handlungsfehler und Misserfolge in der Psychotherapie. Beiträge zur psychosozialen Praxis, Tübinger Reihe Nr. 8. Deutsche Gesellschaft für Verhaltenstherapie, Tübingen, S 73-93

16. Kohn LT, Institute of Medicine (U.S.), Committee on Quality of Health Care in America (2000) To err is human: building a safer health system. National Academy Press, Washington, DC

17. Kottler JA, Blau DS (1991) Wenn Therapeuten irren. Versagen als Chance. Edition Humanistische Therapie, Köln

18. Kuhr A (1988) Fehler und Misserfolge in der Psychotherapie. In: Kleiber D, Kuhr A (Hrsg) Handlungsfehler und Misserfolge in der Psychotherapie. Beiträge zur psychosozialen Praxis, Tübinger Reihe Nr. 8. Deutsche Gesellschaft für Verhaltenstherapie, Tübingen, S 8-17

19. Lehmkuhl U, Lehmkuhl G (2000) Ethische Fragen in der Psychotherapie von Kindern, Jugendlichen und Familien. Ethical questions in the psychotherapy with children, adolescents, and families. Z Individualpsychol 25:184-196

20. Lindenmeyer J (1999) Ethische Fragen in der Verhaltenstherapie. Ethical questions in behavior therapy. In: Tress W, Langenbach M (Hrsg) Ethik in der Psychotherapie. Vandenhoeck \& Ruprecht, Göttingen, S 181-199

21. Lindsay G, Koene C, Ovreeide H, Lang F (2008) Ethics for European psychologists. Hogrefe, Göttingen

22. Margraf J (Hrsg) (1996) Lehrbuch der Verhaltenstherapie, Bd 1: Grundlagen, Diagnostik, Verfahren, Rahmenbedingungen. Springer, Berlin

23. Margraf J, Schneider S (Hrsg) (2009) Lehrbuch der Verhaltenstherapie, Bd 1: Grundlagen, Diagnostik, Verfahren, Rahmenbedingungen. 3., vollst. bearb. u. erw. Aufl. Springer, Berlin

24. Mayring P, Gläser-Zikuda M (Hrsg) (2005) Die Praxis der Qualitativen Inhaltsanalyse. Beltz, Weinheim

25. Medau I, Jox R, Dittmann V, Reiter-Theil S (2012) Eine Pilotstudie zum Umgang mit Fehlern in der Psychotherapie - Therapeuten berichten aus der Praxis. Psychiatr Prax 39:326-331

26. Pateisky N (2004) Fehlerkultur und Teamtraining. Gynäkol 37:73-77

27. Perrow C (1988) Normale Katastrophen. Die unvermeidbaren Risiken der Großtechnik. Campus, Frankfurt

28. Reiter-Theil S (1988) Autonomie und Gerechtigkeit. Das Beispiel der Familientherapie für eine therapeutische Ethik. Springer, Berlin

29. Reiter-Theil S (1996) Informed consent in psychiatry and ethical principles. A comparative analysis. In: Koch HG, Reiter-Theil S, Helmchen H (Hrsg) Informed consent in psychiatry. European perspectives of ethics, law and clinical practice. Nomos, Baden-Baden, S 281-303 
30. Reiter-Theil S, Medau I (2009) Ethische Fragen im Kontext der Verhaltenstherapie. In: Margraf J, Schneider S (Hrsg) Lehrbuch der Verhaltenstherapie, Bd 1. Springer, Berlin, S 249-259

31. Sapyta J, Riemer M, Bickman L (2005) Feedback to clinicians: theory, research, and practice. J Clin Psychol 61:145-153

32. Schleu A, Hillebrand V, Gutmann T (2007) Deutschland. In: Hutterer-Krisch R (Hrsg) Grundriss der Psychotherapieethik. Praxisrelevanz, Behandlungsfehler und Wirksamkeit. Springer, Wien, S 363-399

33. Schmidt-Lellek C, Heimannsberg B (1995) Macht und Machtmissbrauch in der Psychotherapie. Edition Humanistische Psychologie, Köln

34. Sonneck G (2001) Ethische Fragen im Umgang mit Suizidgefährdeten. In: Hutterer-Krisch R (Hrsg) Fragen der Ethik in der Psychotherapie. Springer, Wien, S 307-312

35. Sponsel R (1997) Potentielle Kunst-/Fehler aus der Sicht der Allgemeinen und Integrativen Psychologischen Psychotherapie. Materialien zur Qualitätssicherung mit einer Literaturübersicht. Potential malpractice from the view of general and integrative psychological psychotherapy. Psychol 22:602-604

36. Strupp HH, Hadley SW, Gomes-Schwartz B (1977) Psychotherapy for better or worse: the problem of negative effects. J. Aronson, New York

37. Tress W, Langenbach M (1999) Ethik in der Psychotherapie. Vandenhoeck \& Ruprecht, Göttingen

38. Vetter J (2007) Schweiz. In: Hutterer-Krisch R (Hrsg) Grundriss der Psychotherapieethik. Praxisrelevanz, Behandlungsfehler und Wirksamkeit. Spinger, Wien, S 403-410

39. Wolfslast G (1985) Psychotherapie in den Grenzen des Rechts. Psychotherapy within the boundaries of the law. Medizin in Recht und Ethik, Bd 15. Enke, Stuttgart 